\title{
A Stellarator Reactor based on the Optimization Criteria of Wendelstein 7-X
}

\author{
R. C. Wolf* and the Wendelstein 7-X Team \\ Max-Planck-Institut für Plasmaphysik, Teilinstitut Greifswald, Wendelsteinstraße 1, D-17491 Greifswald, Germany
}

\begin{abstract}
Wendelstein 7-X is an optimized stellarator including a divertor concept for energy and particle exhaust. The main properties of Wendelstein 7-X are discussed in the context of the application to a stellarator fusion reactor - the Helical Advanced Stellarator concept which is based on the same optimization criteria as Wendelstein 7-X. Experience gained from the design, construction of components and the starting assembly of Wendelstein 7-X is presented.
\end{abstract}

Keywords: Stellarator optimization, Wendelstein 7-X, Helical Advanced Stellarator, fusion reactor

\section{Introduction}

The main motivation for the stellarator line is the intrinsically steady state magnetic field. In contrast to the tokamak, the confining magnetic field (i.e. the rotational transform) is to a large extent provided by external coils, thus avoiding (1) the necessity for current drive to achieve steady state plasmas and (2) current driven instabilities or the possibility of disruptions. For a tokamak reactor current drive is still lacking a viable solution, as it is not yet clear which method satisfies, both, the necessary physics (e.g. plasma deposition) and technology requirements (e.g. efficiency to keep the recirculation power low enough). Also the instabilities associated with the strong plasma current are of major concern, and therefore the development of feedback schemes for their avoidance and disruption mitigation techniques are a focus of tokamak research. A disadvantage of a magnetic field, generated by external coils, is that, due to $\int B_{p o l} d l_{p o l}=0$ ( $B_{p o l}$ being the poloidal component of the magnetic field and $\int d l_{p o l}$ the integral along the poloidal circumference), the magnetic field configuration is 3dimensional, i.e. not toroidally symmetric. Both, from an physics and engineering point of view this complicates the design of a stellarator.

Historically the stellarator concept preceded that of the tokamak, dating back to Lyman Spitzer who proposed the stellarator in 1951 to confine hot fusion plasmas [1]. However, the complicated coil geometry, the sensitivity of the $3 \mathrm{D}$ confinement to the details of the coil configuration, and the resulting difficulty to

\footnotetext{
* Corresponding author Tel: +49-(0)3834-882508; fax: +49-(0)3834-882509

E-mail address: robert.wolf@ipp.mpg.de (R. C. Wolf)
} 
make a qualified choice of the optimum configuration prevented early successes. The absence of these complications and, accordingly, an easier achievable confinement resulted in a faster development of the tokamak concept, culminating in the design of ITER as the first burning fusion plasma experiment [2].

Table 1

Characteristics of major stellarators. The ordering of the currents $(\mathrm{O}(1)$ meaning of the order 1$)$ refers to the their respective scale.

\begin{tabular}{|c|c|c|c|}
\hline & W7-X & LHD & NCSX \\
\hline Major radius (m) & 5.5 & $3.6^{1)}$ & 1.5 \\
\hline $\begin{array}{l}\text { Effective minor } \\
\text { radius (m) }\end{array}$ & 0.55 & $0.67^{1)}$ & 0.3 \\
\hline Aspect ratio R/a & 10 & 5.4 & 5 \\
\hline $\begin{array}{l}\text { Superconducting } \\
\text { coils }\end{array}$ & yes & Yes & no \\
\hline Modular coils & yes & no & yes \\
\hline $\begin{array}{l}\text { Development of } \\
\text { steady state } \\
\text { plasma facing } \\
\text { components and } \\
\text { divertor }\end{array}$ & yes & no & $\mathrm{no}^{2)}$ \\
\hline Reactor studies & HELIAS & FFHR & ARIES-CS \\
\hline $\begin{array}{l}\text { Diamagnetic } \\
\text { current }\end{array}$ & $\mathrm{O}(1)$ & $\mathrm{O}(1)$ & $\mathrm{O}(1)$ \\
\hline $\begin{array}{l}\text { Pfirsch-Schlüter } \\
\text { current }\end{array}$ & 0 & $\mathrm{O}(1)$ & $\mathrm{O}(1)$ \\
\hline Bootstrap current & 0 & $\mathrm{O}( \pm 1)^{3)}$ & $\mathrm{O}(1)$ \\
\hline
\end{tabular}

1) Standard configuration

2) Baffle plates and cryo-pumps utilizing the intrinsic helical divertor to form a closed divertor system are in preparation

3) The bootstrap current is positive in the core and negative

The possibility of elaborate numerical optimization procedures opened the path to design quasi-symmetric magnetic field configurations which overcome the problems of the 3D geometry. Consequently, basic reactor properties such as good magnetic surfaces, favourable equilibrium properties and magnetohydrodynamic stability at sufficiently high normalized plasma pressure, $\beta$, and satisfactory thermal and fast particle confinement should be achievable. Besides Wendelstein 7-AS, which was partially optimized with respect to reduced equilibrium currents [3], recent experimental results confirm the effectiveness of quasisymmetry regarding improved neoclassical confinement [4].

Within the family of optimized stellarators the quasi-isodynamic Wendelstein 7-X (W7-X) design is based on the minimization of all internal plasma currents (except the diamagnetic current) [5], while the quasi-toroidal National Compact Stellarator Experiment (NCSX) relies on a large bootstrap current to generate the desired rotational transform [6]. Table 1 compares these two devices, which are presently under construction, with the Large Helical Device (LHD), a classical stellarator with superconducting coils, which went into operation in 1998 [7]. For all three types of devices reactor studies have been performed, analysing the different concepts with respect to their performance as fusion reactors, namely the Force Free Helical Reactor, FFHR [8], the ARIES Compact Stellarator, ARIES-CS [9], and the HElical Advanced Stellarator, HELIAS [10, 11]. All three device concepts are helical systems with an intrinsically steady state magnetic field. As NCSX and W7-X, ARIES-CS and HELIAS both employ modular coils, but follow different optimization schemes resulting in quite different aspect ratios R/a (see table 1). In contrast, the FFHR design has continuous helical coils with the advantage of low forces in the helical windings enabling a simplified support structure and thus wide maintenance ports.

In the following this paper focuses on the HELIAS reactor considering the experience gained from the design, construction of components and starting assembly of W7-X.

\section{Optimization criteria of Wendelstein 7-X}

The aim was to design a magnetic field configuration minimizing internal plasma currents, so that the equilibrium is provided by external magnetic field coils only and also the plasma pressure has only a weak influence. The magnetic field configuration of W 7-X has five field periods and low magnetic shear, with a rotational transform, $l$, in the vicinity of 1 . The $\imath$ range covers configurations with four (high $l=1.2$ ), five (standard case at $l=1$ ) and six magnetic islands (low $l=0.8)$ at the plasma edge. The magnetic field is realized with 50 modular coils (10 per field period) and 20 planar coils for higher experimental flexibility (i.e. plasma position control). The whole device is designed for a discharge duration of 30 minutes which includes superconducting coils, an actively cooled divertor and an electron cyclotron resonance heating (ECRH) system which can deliver up to $10 \mathrm{MW}$ over such a 


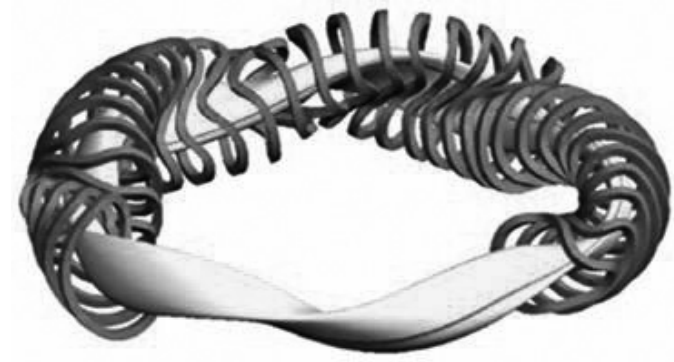

Fig. 1. Schematic of the modular coils system of W7-X, also showing the plasma surface with one magnetic field line (the planar coils for position control of the plasma are not shown).

time period [12].

The optimization criteria after which $\mathrm{W} 7-\mathrm{X}$ is designed and constructed are discussed in the following.

\subsection{Feasible modular coils}

Modular coils are discrete coils which provide both poloidal and toroidal magnetic field components, producing nested magnetic surfaces with multiple helicity [13]. The modular coil arrangement of W7-X is shown in Fig. 1.

The advantage of modular coils is the possibility to adapt the coils system to the requirements of the

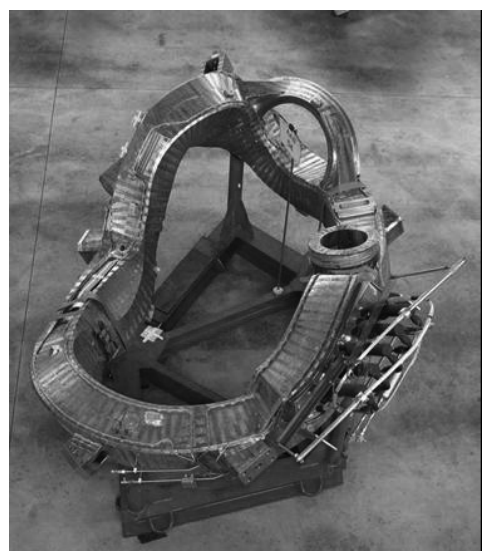

Fig. 2. Non-planar coil of W7-X (AAB54) mounted on a transport frame. specified magnetic field. In this procedure, first the magnetic field is optimized according to the optimum plasma performance. Subsequently the coil system, which generates this magnetic field, is computed. In addition, modular coils realize a helical magnetic field without the need of helically continuous coil windings which are of the size of the device itself. Thus, they can be manufactured individually, separating the coil manufacturing process from the machine assembly. However, their non-planar shape (Fig. 2), results in an inhomogeneous force distribution with radial, lateral and vertical components [14].

\subsection{Good nested magnetic surfaces}

Good magnetic surfaces of the vacuum magnetic field without major resonances, islands or stochastic regions in the plasma core are essential for good magnetic confinement. Only recently evidence was found that indeed the $\beta$-limit in W7-AS is caused by a increasing stochastization of magnetic field lines, resulting in a rising field line diffusion in the outer third of the plasma cross-section [15]. In the plasma boundary islands or stochastic regions can be tolerated or even used as a divertor for the controlled particle and power exhaust.

In particular for a low shear stellarator such as W7-X operating near a rotational transform of $l=1$, deviations from the ideal coil geometry result in resonant perturbations which eventually destroy the islands at the boundary envisaged for divertor action. For the construction of W7-X this means that the deviation of the coil conductors from the ideal contour must be kept within the repetition tolerances of a few millimetres [16]. Measurements of the shape of the manufactured coils show average deviations of the central filament position from the design shape of less than $3 \mathrm{~mm}$ [17]. For the compensation of the additional anticipated assembly errors correction coils (in form of saddle coils) can be used [18]. To avoid these problems in a HELIAS reactor one may choose $t=5 / 6$ or $l=6 / 5$ at the boundary, where the islands are not independent and therefore less vulnerable to field perturbations.

\subsection{Stiff equilibrium configuration}

In toroidal equilibria the shift of the inner magnetic 


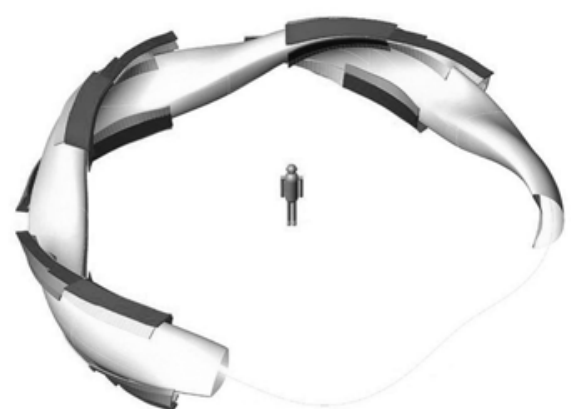

Fig. 4. Schematic of the W7-X divertor, showing target plates (red), baffles (yellow), and the boundary of the plasma core (white).

surfaces with rising plasma pressure is called Shafranov shift. By keeping the Pfirsch-Schlüter currents small, the Shafranov shift is minimized and thus the magnetic field configuration becomes nearly invariant to changes of the plasma pressure. For W7-X the equilibrium should exhibit only minor modification up to an average $\beta$ of $5 \%$.

The main reason for this kind of optimization is the implementation of a viable divertor concept. Provided the size and, in particular, the position of the islands do not change too much with variations of the pressure, the five magnetic islands at the plasma boundary can be utilized to interface the plasma core
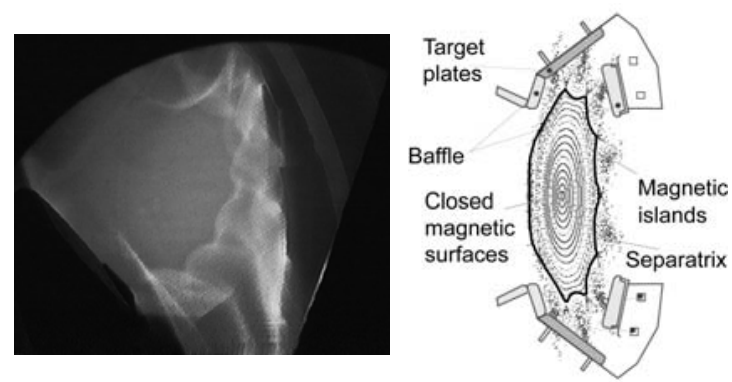

Fig. 3. Left: Toroidal view of the (poloidal) plasma crosssection of an island divertor plasma of W7-AS. The bright emission corresponds to cold plasma in the island divertor region. Right: Poloidal cross-section, showing the closed magnetic surfaces of the plasma core, separated from the magnetic islands and regions with open magnetic field lines by the separatrix, and target plates and baffles of the divertor structure. with high heat flux target plates. In contrast to a tokamak with a poloidal divertor, the magnetic field structure of the island divertor results from the resonance condition $(l=1)$ at the plasma boundary. Therefore the target plates have to be carefully aligned with the island chains, helically winding around the confined plasma core (Fig. 4).

The first experiment to verify this concept was W7-AS [19]. Fig. 3 shows the poloidal cross-section of the plasma with the closed magnetic surfaces in the core and the open field lines of the magnetic islands intersecting with the target plates. The corresponding image shows the (cold) plasma radiation following the island divertor topology.

\subsection{Good magneto-hydro-dynamic (MHD) stability}

Sufficiently high plasma pressure is a prerequisite for high fusion power density. Besides the above mentioned equilibrium limit, $\beta$ is limited by the onset of MHD instabilities.

Without strong plasma currents, tearing modes or even disruptions are absent in stellarators. Relevant instabilities which remain are resistive interchange and ballooning modes (ideal and resistive) [5]. Since in the W7-X configuration the magnetic shear is small, the stability of such modes relies in particular on a sufficiently strong magnetic well. Finally, in finite $\beta$ plasmas low order rational surfaces can occur, which in the vacuum magnetic field do not exist, resulting in the formation of global modes [20]. Overall a $\langle\beta\rangle \approx 5 \%$ is anticipated for W7-X.

\subsection{Small neoclassical transport}

Trapped particle orbits together with Coulomb collisions can lead to strong radial losses of these particles. This affects both the thermal plasma, reducing the triple product, and fast particles, the confinement of which is required for the internal heating of the fusion plasma. Owing to the existence of a helical ripple in a 3D geometry, superimposed to the toroidal ripple of an axisymmetric configuration, the neoclassical transport is much larger for the $1 / v$ regime (i.e. low collisionality) in stellarators than in tokamaks [21]. To guarantee sufficiently low neoclassical confinement in the $1 / \mathrm{v}$ regime, the effective helical ripple must not exceed $2 \%$. 
Modular coils introduce also a ripple. The associated radial drifts, due to particles trapped in the modular ripple well, can only be kept smaller than the remaining helical ripple losses if the number of modular coils is high enough. For W7-X this results in a condition asking for at least ten coils per field period.

\subsection{Small bootstrap current}

Another neoclassical effect is the bootstrap current, a toroidal current, which is driven by the anisotropic pressure of the plasma. Since the anisotropy grows at low collisionality with increasing of the mean free path of the particles, this current becomes large in reactor relevant regimes. In particular in low shear stellarators the associated change of $\imath$ may result in an undesired overlap of major resonances. The W7-X optimization could achieve a reduction by a factor of about 20, which implies that the bootstrap current is only $5 \%$ of that in a equivalent axisymmetric device. This is in contrast to the quasitoroidal configuration of NCSX where the bootstrap current forms an essential part of the rotational transform [6].

Experiments in tokamaks and stellarators have confirmed the theory of the bootstrap current. In W7AS, which was not optimized with respect to low bootstrap currents, theory and experiment agreed well [22], giving confidence for the optimization of W7-X.

\subsection{Good confinement of fast particles}

In a fusion reactor sufficient confinement of the highly energetic $\alpha$-particles is necessary for a self sustained burning fusion plasma. In addition,

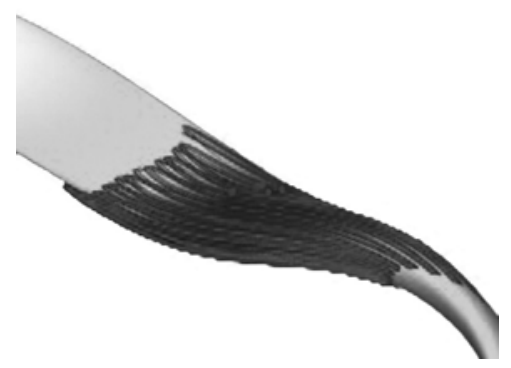

Fig. 5. Poloidally closed drift orbit (here for a $300 \mathrm{keV}$ triton) trapped in the helical mirror of W7-X [24]. (localized) losses of energetic particles to the wall risks serious damage to wall components.

In stellarators the basic problem of fast particle confinement is the lack of continuous symmetry, generally resulting in a radial drift of these particles as they are hardly affected by collisions or radial electric fields [23]. To transfer their energy to the thermal plasma, the $3.5 \mathrm{MeV} \alpha$-particles should stay in the plasma for at least one slowing down time, which in a fusion reactor would be of the order of $100 \mathrm{msec}$.

The quasi-isodynamic symmetry of W7-X solves this problem by increasing the magnetic field in transition areas between the five field periods, basically establishing a system of linked mirrors [24]. The trapped particles oscillate between these regions of high magnetic field, making a net poloidal rotation but no radial movement, as they are kept away from zones of high field inhomogeneity (Fig. 5). A particular characteristic of the W7-X configuration is that the collisionless fast particle confinement requires a finite $\beta$ of about $2-3 \%$ [23].

Besides the verification of the optimization criteria, further objectives of W7-X are to explore the operational space with respect to the optimum triple product, $n T \tau_{E}$, verify $\beta$-limiting effects, demonstrate steady state operation including the island divertor concept, use the steady state capability for plasma wall interaction studies, and investigate the influence of the optimized stellarator configuration on turbulent transport. To this end the $10 \mathrm{MW}$ ECRH will be augmented at a later stage by $20 \mathrm{MW}$ of neutral beam injection and $2 \mathrm{MW}$ of ion cyclotron resonance heating.

Regarding high density operation a particularly interesting feature of stellarators apparently is the absence of a density limit, which is favourable for achieving high $n T \tau_{E}$ at the optimum temperature of about $15 \mathrm{keV}$. While in tokamaks the Greenwald limit relates the achievable density to the plasma current and the current is limited for stability reasons, in stellarators no hard limits have been observed. Although too high density gradients together with good neoclassical confinement show the tendency for impurity accumulation, ultimately leading to a radiation impurity collapse, regimes such as the high density H-mode in W7-AS [25] or the superdense core mode in LHD [26] have been found which sustain 
densities of several $10^{20} \mathrm{~m}^{-3}$, well beyond the Greenwald limit of tokamaks [27]. It, however, remains to be seen how these regimes extrapolate to plasmas with higher temperatures and lower collisionality.

\section{The HELIAS Reactor}

The HELIAS reactor (HSR) design studies are an extrapolation from the W7-X design to a reactor size stellarator [10, 11, 28]. In particular the chosen quasisymmetry and the optimization procedure are the same,

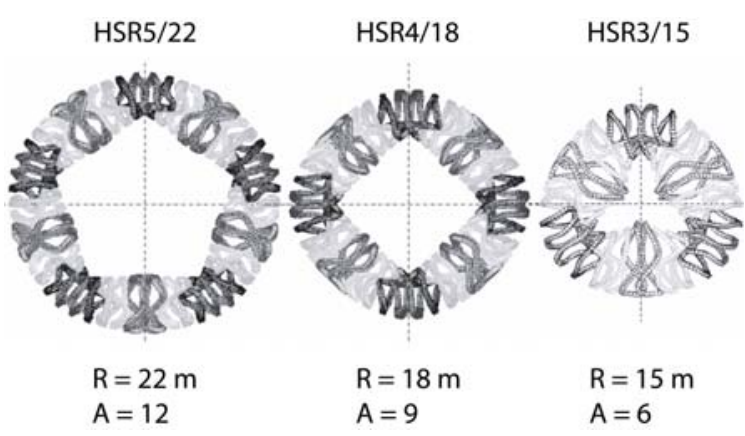

Fig. 6. Top view of the modular coils for the different HELIAS reactor studies. The colors indicate repeating coils types.

decoupling as much as possible plasma and magnetic field properties.

Three different reactor configurations have been investigated: (i) The HSR5/22, a scaled up version of W7-X, with five field periods, a major radius of $\mathrm{R}=22 \mathrm{~m}$ and an aspect ratio of $\mathrm{A}=12$, (ii) the HSR4/18 with four field periods, $18 \mathrm{~m}$ major radius and a reduced aspect ratio of $A=9$ [10], and finally the HSR3/15 with only three field periods, $R=15 \mathrm{~m}$ and an even further reduced aspect ration of $A=6$ (Fig. 6). Reducing the aspect ratio fewer coils are needed, however at the expense of an increasing the modular ripple and the associated fast particle losses. While the first two configurations have been studied in many details, the HSR3/15 still needs optimization of the magnetic field, such as minimization of the bootstrap current, improvement of $\alpha$-particle confinement, and verification of an acceptable modular field ripple. As the minor radius increases with decreasing major radius, the plasma volume is similar for all three configurations. Also the average magnetic field (on axis about $5 \mathrm{~T}$ or slightly below which is twice that of $\mathrm{W} 7-\mathrm{X})$, the aspired $\beta(4-5 \%)$ and hence the fusion power (3 GW) are comparable.

\subsection{Additional physics issues}

The assumed confinement quality and the required blanket thickness basically determine the size (i.e. the volume) of the reactor. The required confinement time is in the range of 2.5 to $3 \mathrm{sec}$. Unfortunately a unified scaling law, covering existing stellarator experiments, is still an open issue. The Lackner-Gottardi scaling and scalings derived from the W7 experiments and LHD even predict larger confinement times. Hence, no improvement factors or $\mathrm{H}$-mode confinement has been assumed to reach the ignition condition. Although still preliminary, latest scalings improve the cross-device fit, suggesting a configuration factor which appears to correlate with the degree of neoclassical optimization [29]. Altogether the question is how the anomalous (turbulent) transport depends on the configuration, which comprises the relation between anomalous and neoclassical transport drives, the scaling of favourable confinement regimes with normalized plasma parameters (normalized gyro-radius, collisionality and $\beta$ ), and turbulent impurity transport.

A further issue is the divertor behaviour in reactor relevant regimes. At the high densities, which can be achieved by stellarators, impurity control will become crucial. In addition, the larger aspect ratio and larger first wall surface (compared to a tokamak) may increase plasma recycling and impurity sources. In particular, for a feasible route to ignition during the initial phase of high density and low temperature, when impurity ions easily accumulate in the centre, a radiation collapse has to be avoided.

Also the questions relating to the choice of first wall material are still open. Due to the high tritium retention and strong erosion, carbon is considered as not acceptable for a fusion reactor. The presently favoured alternative material is tungsten. Already in ITER, carbon is presently foreseen only for the most heavily loaded areas in the divertor. To test this concept, JET will be equipped with an ITER like first wall consisting of beryllium in the main chamber and tungsten in the divertor, giving priority to a divertor even without any carbon [30]. While in the ASDEX Upgrade tokamak the first results with a tungsten 
covered first wall look promising [31], the stronger tendency for impurity accumulation in a stellarator may make this question more difficult to solve. Therefore, W7-X will start its steady state operation phase with actively cooled divertor and baffle target plates covered with carbon.

Finally, besides the neoclassical optimization of the collisionless fast particle confinement, a burning fusion plasma is characterized by a significant fast particles population. These $\alpha$-particles can induce collective instabilities (Alfvén eigenmodes) which may increase the fast particle losses. Theoretical studies of the Alfvén continuum and high frequency eigenmodes with application to HELIAS configurations have been published by Kolesnichenko et al. [32]. One of the important tasks of ITER will be to study these effects. The extrapolation to a stellarator reactor will have to take the 3D magnetic field geometry and possible synergies with the 3D-orbit losses into account [e.g. 33].

\subsection{Engineering issues and experience from $W 7-X$ construction}

Important engineering issues related to a fusion reactor are the superconducting coils, the coil support structure, high heat flux components such as the divertor, the breeding blanket and neutron shield, and accessibility for maintenance.

One of the main cost-driving components in a fusion reactor will be the coils. On the other hand, the limits from plasma physics require high magnetic field for good confinement and moderate $\beta$. The advantage of the HELIAS design is that an average field on the magnetic axis of only $5 \mathrm{~T}$ and, correspondingly, a maximum magnetic field at the coils of $10 \mathrm{~T}$ appears to be sufficient [28]. As a consequence, for all coils NbTi can be used as superconductor material. However, for $10 \mathrm{~T}$ this requires superfluid helium for cooling down to $1.8 \mathrm{~K}$. Alternatively, $\mathrm{Nb}_{3} \mathrm{Al}$ has been proposed as it combines a higher critical field with good mechanical properties. Whether the $\mathrm{Nb}_{3} \mathrm{Sn}$ super-conductor, which will be used for part of the ITER coils [34], is also an option still needs to be investigated, especially in view of the application of the "wind and react" method in large modular coils. At the lower magnetic field of W7-X (3.0 T on axis and 6.8 T maximum field at some places of the coils), the coolant temperature for $\mathrm{NbTi}$ can be relaxed to about $4 \mathrm{~K}$. W7-X uses a cable-in- conduit conductor, made of NbTi strands covered with copper and embedded in an jacket of aluminium alloy. This type of conductor was chosen to meet the contradictory requirements of, on the one hand, easy winding of the complex coil shapes (with almost not spring-back) and, on the other hand, high mechanical strength at operation [35, 36]. In the structural analysis of the HELIAS reactor coil system performed up to now the cable-in-conduit conductor is derived from the W7-X design [14] (using NbTi at 1.8 K). Problems to reproducibly fabricate high quality welds of the aluminium jackets of the W7-X conductors suggest that alternative materials such as steel should also be considered in the future. Up to now all tested W7-X coils confirmed the predicted conductor performance, even with less degradation during coil production than expected [37]. One of the most critical problems of the W7-X coils were faults of the electrical insulation, mainly in the header or joint areas. It turned out that high voltage tests under Paschen conditions, sometimes also in combination with cold tests (for thermal cycling), were indispensable to detect these faults $[38,39]$. From this experience is advisable to test the coils of any future super-conducting device. The advantage of the HELIAS design is that individual coils can be tested in a vessel which still has a manageable size. For comparison, the inner diameter of the HSR4/18 cryostat is $20 \mathrm{~m}$, the largest outer diameter of the coils is about $14 \mathrm{~m}$.

In a stellarator with modular coils the coil support structure needs particular attention. Although electromagnetic forces due to disruptions do not exist, the non-planar shape of the coils requires a rather complex mechanical structure to support the forces generated by the interaction of the coil currents with the magnetic field they generate [40]. In W7-X the coils are suspended on a central support ring with support elements between them. Depending on available space and the accessibility during assembly, different types of connections have been chosen (bolts, welded connections or sliding elements) [41]. The challenge for such a structure is to combine the necessary load capacity with the alignment accuracy of the coils in the range of a few millimetres. An additional worry, related to the cable-in-conduit superconductor, is that stick-slip in moving mechanical components, such as the so-called narrow support elements, during energising the coils may induce quenches in the superconductors [42, 43]. The narrow 
support elements are placed between coils to take up the orthogonal forces, but are essentially free to move in the lateral directions. Similar to W7-X the HSR design is based on rigid coil housings and the mutual support of the coils forming toroidal and helical rings [44].

The heat and particle flux leaving the plasma is to a large extent deposited on the divertor. For the HELIAS reactor the same divertor topology as in $\mathrm{W} 7-\mathrm{X}$ is foreseen. Looking simply at the heat flux through the outer flux surface, for $10 \mathrm{MW}$ of heating power the $0.1 \mathrm{MW} / \mathrm{m}^{2}$ of $\mathrm{W} 7-\mathrm{X}$ is a factor of four below the HSR5/22 and HSR4/18 values of about $0.4 \mathrm{MW} / \mathrm{m}^{2}$. Assuming only convective transport along the open field lines without any plasma radiation this leads to steady state heat fluxes in the divertor of W7$\mathrm{X}$ locally of up to $10 \mathrm{MW} / \mathrm{m}^{2}$. The pre-series water cooled target elements achieved this value even with some safety margin [45]. To stay below the limit of $10 \mathrm{MW} / \mathrm{m}^{2}$ in the HELIAS design up to $90 \%$ of the alpha-particle power has to be radiated, requiring detached divertor operation and high separatrix densities. Because of the aforementioned issue of impurity control in stellerators in general, the question of amour materials and their compatibility with plasma operation still has to be solved. In general, the coolant requirements for the HELIAS divertor is similar to other reactor concepts (stellarator or tokamak). However, dedicated studies have not yet been conducted.

In a fusion reactor the blanket is required for breeding tritium and protecting the coils from radiation damage and large nuclear heating. As a reminder, the derived blanket thickness and the assumed confinement quality determine the size of the reactor. In the HELIAS design $1.3 \mathrm{~m}$ as the closest distance between plasma and coils is regarded as sufficient to achieve these goals. In this context an advantage of the HELIAS - compared to a tokamak - is the larger aspect ratio which reduces the neutron wall flux and hence increases the lifetime of the wall components, the structural material and the blanket. Computations of the $14 \mathrm{MeV}$ neutron emission by the plasma show a strongly inhomogeneous wall loading with an average level of less than $1 \mathrm{MW} / \mathrm{m}^{2}$ and peak values of about 1.6 $\mathrm{MW} / \mathrm{m}^{2}$ [28]. Blanket options envisaged are the helium cooled solid breeder blanket or the water cooled $\mathrm{Li}$-Pb blanket. In addition, the absence of disruptions eliminates sudden electro-mechanical loads and excessive transient heat fluxes. However, the 3D geometry of the blanket system and the need of maintenance through ports, the maximum size of which is determined by the distance between the modular coils, results in a large number of blanket elements. Owing to the complex 3D geometry, already the vacuum vessel and the thermal insulation inside the cryostat of W7-X required specific development work. The thermal insulation, for instance, was a completely new development, as it has to cover a non-developable surface with a limited thickness which has to meet narrow tolerances [46].

\section{Summary}

The main advantage of stellarators - compared to tokamaks - is their steady state magnetic field and the absence of current driven instabilities and disruptions. In a fusion reactor this means lower recirculating power. In addition, a hard density limit does not seem to exist. The task of W7-X is to verify the employed optimization criteria with respect to their applicability to a HELIAS reactor, including steady state divertor operation and suitable confinement regimes at relevant plasma parameters. Advantages of the HELIAS concept are the prospects of good $\alpha$-particle confinement and the integration of a divertor in the optimization procedure. The relatively large aspect ratio eases the requirements for the blanket design. However, issues are the tendency for impurity accumulation and, related to the complex 3-D geometry, the necessary precision of the fabrication and assembly of coils and coil support structure. To alleviate the latter problem in a HELIAS reactor, one could choose $\imath$ slightly above or below one where the corresponding magnetic islands are less vulnerable to magnetic field perturbations.

\section{References}

[1] Spitzer L Jr US Atomic Energy Commissioning Report No. NYO-993 (PM-S-1), 1951 and Spitzer L Jr Phys. Fluids 1 (1958) 253

[2] Aymar R et al. Nucl. Fusion 41 (2001) 1301

[3] W7-AS review, submitted to PPCF

[4] Canik J M et al. Phys. Rev. Lett 98 (2007) 085002

[5] Grieger G at al. Phys. Fluids B4 (1992) 2081 
and

Beidler C et al. Fusion Techn. 17 (1990) 148

[6] Zarnstorff $\mathrm{M}$ et al. Plasma Phys. Control Fusion 43 (2001) A237

[7] Motojima O et al. Nucl. Fusion 45 (2005) S255

[8] Sagara A et al. Nucl. Fusion 45 (2005) 258

[9] Najmabadi F, Raffray A R, and the Aries Team Fusion Eng. Design 81 (2006) 2679

[10] Beidler C D et al. Nucl. Fusion 41 (2001) 1759

[11] Andreeva et al. Fusion Science and Technology 46 (2004) 395

[12]Erckmann et al. Fusion Science and Technology 52 (2007) 291

[13] Wobig W and Rehker S Proc. $7^{\text {th }}$ Symp. on Fusion Techn., Grenoble, France (October 1972) 333

[14] Harmeyer E and Kisslinger J Fusion Eng. Design 66-68 (2003) 1025

[15]Reiman A and Zarstorff M et al. Nucl. Fusion 47 (2007) 572

[16] Andreeva $\mathrm{T}$ et al. Fusion Science and Technology 46 (2004) 388

[17] Andreeva T and Kisslinger J Fusion Science and Technology 50 (2006) 258

[18] Kisslinger J and Andreeva T Fusion Science and Technology 50 (2006) 382

[19] Grigull P et al. Plasma Phys. Control. Fusion 43 (2001) A175

[20] Nührenberg C Phys. Plasmas 3 (1996) 2401

[21]Drevlak M et al. Proc. 30th EPS Conference on Contr. Fusion and Plasma Phys., St. Petersburg, 7-11 July 2003 ECA Vol. 27A, P-1.12

[22] Wagner F et al. Phys. Plasmas 12 (2005) 072509

[23] Lotz W, Merkel P, Nührenberg J, and Strumberger E Plasma Phys. Control Fusion 34 (1992) 1037

[24] Strumberger E Nucl. Fusion 40 (2000) 1697

[25] McComick K et al. Phys. Rev. Lett. 89 (2002) 015001

[26] Morisaki T et al. Phys. Plasmas 14 (2007) 056113

[27]Weller A et al. Plasma Phys. Control. Fusion 45 (2003) A285

[28] Igitkhanov Yu et al. Fusion Eng. Design 81 (2006) 2695

[29]Dinklage A et al. Fusion Science Techn. 51 (2007) 1

[30] Pamela J et al. Fusion Eng. Design 363-365
(2007) 1

[31]Neu R et al. Fusion Eng. Design 363-365 (2007) 52

[32] Kolesnichenko Ya et al. Phys. Plasmas 8 (2001) 491

[33] Kolesnichenko Ya et al. Phys Plasmas 9 (2002) 517

[34] Martovetsky N Physica C 401 (2004) 22

[35] Risse K et al. Fusion Eng. Design 66-68 (2003) 965

[36] Maix R K et al. Journal of Phys.: Conf. Series 43 (2006) 753

[37]Rummel Th, Risse K, Ehmler H Fusion Eng. Design 75-79 (2005) 117

[38] Viebke $\mathrm{H}$ et al. Journal of Phys.: Conf. Series 43 (2006) 748

[39] Scheller H et al. IEEE Transactions on Applied Superconductivity 16 issue 2 (2006) 759

[40] Harmeyer E et al. Fusion Eng. Design 58-59 (2001) 265

[41] Gasparotto M et al.Fusion Eng. Design 74 (2005) 161

[42] Haange R and the W7-X Team Proc. 21st IAEA Fusion Energy Conference (October 2006) Chengdu, China, FT/2-3

[43] Schauer F, IEEE Transactions on Magnetics 32 (1996) 2760

[44] Wobig H et al. Nucl. Fusion 43 (2003) 889

[45] Greuner H et al. Phys. Scr. T128 (2007) 218

[46] Schauer F et al. Fusion Eng. Design 56-57 (2001) 861 